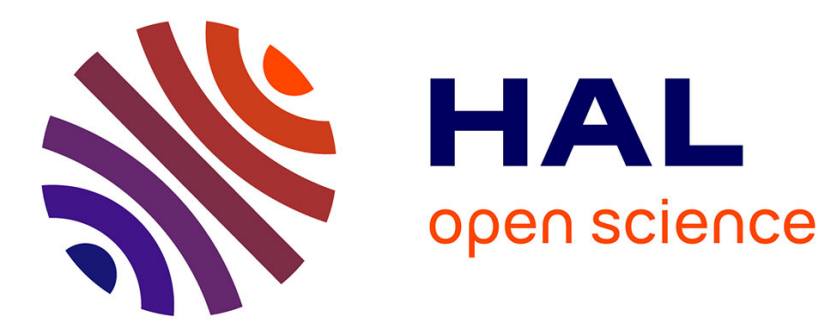

\title{
Interpreting the iISS Small-Gain Theorem as Transient Plus ISS Small-Gain Regulation
}

\author{
Hiroshi Ito, Randy Freeman, Antoine Chaillet
}

\section{To cite this version:}

Hiroshi Ito, Randy Freeman, Antoine Chaillet. Interpreting the iISS Small-Gain Theorem as Transient Plus ISS Small-Gain Regulation. 49th IEEE Conference on Decision and Control, Dec 2010, Atlanta, United States. 6 p. hal-00522198

\section{HAL Id: hal-00522198 \\ https://hal-centralesupelec.archives-ouvertes.fr/hal-00522198}

Submitted on 30 Sep 2010

HAL is a multi-disciplinary open access archive for the deposit and dissemination of scientific research documents, whether they are published or not. The documents may come from teaching and research institutions in France or abroad, or from public or private research centers.
L'archive ouverte pluridisciplinaire HAL, est destinée au dépôt et à la diffusion de documents scientifiques de niveau recherche, publiés ou non, émanant des établissements d'enseignement et de recherche français ou étrangers, des laboratoires publics ou privés. 


\title{
Interpreting the iISS Small-Gain Theorem as Transient Plus ISS Small-Gain Regulation
}

\author{
Hiroshi Ito, Randy A. Freeman and Antoine Chaillet
}

\begin{abstract}
This paper addresses the problem of establishing stability of interconnections of integral input-to-state stable (iISS) systems. Recently, the small-gain theorem for input-tostate stable (ISS) systems has been extended to the class of iISS systems. It has been also proved that at least one of the two iISS subsystems comprising a feedback interconnection needs to be ISS with respect to the state of the other subsystem for guaranteeing the iISS of the overall system. This paper shows that making use of this necessary condition enables to provide more insight on the iISS small gain theorem by giving an alternative proof of this result from the perspective of transient plus ISS small-gain regulation.
\end{abstract}

\section{INTRODUCTION}

The ISS small-gain theorem has been widely used in analysis and design of nonlinear systems [11], [16]. The theorem deals with input-to-state stable (ISS) systems and answers the question of whether their feedback interconnection is again ISS. It was first proved with a trajectory-based approach in [11]. A version relying on the Lyapunov functions associated to each of the subsystems was subsequently presented in [10]. While the construction of a Lyapunov function for the overall interconnection is useful from the analysis and design viewpoints, the trajectory-based proof is simpler and illustrates more intuitively the idea of contraction. Recently, the small-gain theorem has been extended to the interconnection of integral input-to-state stable (iISS) systems in [6], [9]. The iISS is a more general robustness property than ISS [13], [14], and the theorem in [6], [9] includes the ISS small-gain theorem as a special case. In these references, a Lyapunov function is explicitly constructed for the overall interconnection. Another approach, developed in [1], makes use of monotonicity and nullclines in deriving the small gain condition to guarantee that the interconnection of iISS systems is globally asymptotically stable (GAS). Although the approach proposed there does not apply to systems with exogenous inputs, it offers a unified interpretation of GAS for interconnections of iISS and ISS subsystems.

The purpose of this paper is to elucidate the iISS smallgain mechanism with and without external signals. In other words, this paper gives a trajectory-based interpretation

The work is supported in part by Grant-in-Aid for Scientific Research of JSPS under grant 19560446 and 22560449.

$\mathrm{H}$. Ito is with the Department of Systems Design and Informatics, Kyushu Institute of Technology, 680-4 Kawazu, Iizuka 820-8502, Japan hiroshi@ces. kyutech.ac.jp.

R.A. Freeman is with the Department of Electrical Engineering and Computer Science, Northwestern University, Evanston, IL 60208-3118 USA freemandeecs. northwestern. edu.

A. Chaillet is with L2S, EECI, Univ. Paris Sud 11 - Supélec, 3 rue Joliot Curie, 91192 Gif-sur-Yvette, France chaillet@ieee.org. which connects the iISS small-gain theorem with the contractive behavior of trajectories explained by the ISS smallgain theorem. In order to understand how the trajectories of interconnected systems evolve, this paper assumes that iISS dissipation inequalities are given for both individual subsystems. As illustrated by the result in [5] on cascaded iISS systems, the use of dissipation inequalities of subsystems is more successful than using trajectory bounds when dealing with interconnected iISS systems. This paper follows this idea to tackle feedback interconnected systems. The proof this paper develops splits the system trajectory into a transient response and a subsequent response governed by the ISS small-gain condition. This paper illustrates how this strategy can be made possible for dealing with iISS systems which are not ISS.

The following notation is used throughout this paper. The symbol $|\cdot|$ stands for the Euclidean norm. A continuous function $\omega: \mathbb{R}_{+}:=[0, \infty) \rightarrow \mathbb{R}_{+}$is said to be positive definite and denoted by $\omega \in \mathcal{P}$ if it satisfies $\omega(0)=0$ and $\omega(s)>0$ holds for all $s>0$. A function is of class $\mathcal{K}$ if it belongs to $\mathcal{P}$ and is strictly increasing; of class $\mathcal{K}_{\infty}$ if it is of class $\mathcal{K}$ and is unbounded. The identity map on $\mathbb{R}$ is denoted by Id. For a function $\gamma \in \mathcal{P}$, we write $\gamma \in \mathcal{O}(>L)$ with a non-negative number $L$ if there exists a positive number $K>L$ such that $\limsup _{s \rightarrow 0+} \gamma(s) / s^{K}<\infty$ holds. We write $\gamma \in \mathcal{O}(L)$ when $K=L$. The symbols $\vee$ and $\wedge$ denote logical sum and logical product, respectively. For $f, g$ : $\mathbb{R}_{+} \rightarrow \mathbb{R}_{+}$, we use the simple notation $\lim f(s)=\lim g(s)$ to describe $\{\lim f(s)=\infty \wedge \lim g(s)=\infty\} \vee\{\infty>$ $\lim f(s)=\lim g(s)\}$. Note that the $\infty$ case is included. In a similar manner, $\lim f(s) \geq \lim g(s)$ denotes $\{\lim f(s)=$ $\infty \vee \infty>\lim f(s) \geq \lim g(s)\}$. A system $\dot{x}=f(x)$ admitting a unique maximal solution $x(t) \in \mathbb{R}^{n}$ for any initial condition $x(0) \in \mathbb{R}^{n}$ is said to be GAS if its origin is globally asymptotically stable. We let $\mathcal{U}$ denote the set of all measurable locally essentially bounded signals $u: \mathbb{R}_{+} \rightarrow$ $\mathbb{R}^{m}$. A system $\dot{x}=f(x, u)$ admitting a unique solution $x(t)$ on $\mathbb{R}^{n}$ for any initial condition $x(0) \in \mathbb{R}^{n}$ and any $u \in \mathcal{U}$ is said to have the Bounded Energy Frequently Bounded State (BEFBS, [2]) property with respect to input $u$ and state $x$ if there exists $\sigma \in \mathcal{K}_{\infty}$ such that, if $\int_{0}^{\infty} \sigma(|u(\tau)|) d \tau<\infty$ then $\liminf _{t \rightarrow \infty}|x(t)|<\infty$ for all initial conditions $x(0)$.

A preliminary result of this paper was presented in [8].

\section{A REVIEW OF iISS SMALL-GAIN THEOREM}

Consider the following interconnected system:

$$
\Sigma:\left\{\begin{array}{l}
\Sigma_{1}: \dot{x}_{1}=f_{1}\left(x_{1}, x_{2}, r_{1}\right) \\
\Sigma_{2}: \dot{x}_{2}=f_{2}\left(x_{1}, x_{2}, r_{2}\right)
\end{array}\right.
$$


where $x_{i}(t) \in \mathbb{R}^{n_{i}}, r_{i}(t) \in \mathbb{R}^{m_{i}}, x=\left[x_{1}^{T}, x_{2}^{T}\right]^{T} \in \mathbb{R}^{n}$ and $r=\left[r_{1}^{T}, r_{2}^{T}\right]^{T} \in \mathbb{R}^{m}$. In addition to the existence of a unique maximal solution $x(t)$ for any initial condition $x(0) \in \mathbb{R}^{n}$ and any measurable, locally essentially bounded external input $r$, we assume that the two subsystems satisfy the following dissipation inequalities:

Assumption 1: For each $i \in\{1,2\}$, there exist a continuously differentiable, positive definite and radially unbounded function $V_{i}: \mathbb{R}^{n_{i}} \rightarrow \mathbb{R}_{+}$and class $\mathcal{K}$ functions $\alpha_{i}, \sigma_{i}, \sigma_{r i}$ such that

$$
\begin{aligned}
& \dot{V}_{1}\left(x_{1}\right) \leq-\alpha_{1}\left(V_{1}\left(x_{1}\right)\right)+\sigma_{1}\left(V_{2}\left(x_{2}\right)\right)+\sigma_{r 1}\left(\left|r_{1}\right|\right) \\
& \dot{V}_{2}\left(x_{2}\right) \leq-\alpha_{2}\left(V_{2}\left(x_{2}\right)\right)+\sigma_{2}\left(V_{1}\left(x_{1}\right)\right)+\sigma_{r 2}\left(\left|r_{2}\right|\right)
\end{aligned}
$$

hold for all $r \in \mathcal{U}$ along the trajectories $x(t)$ of (1).

This assumption imposes that each subsystem $\Sigma_{i}$ is iISS with respect to input $\left(x_{3-i}, r_{i}\right)$ and state $x_{i}$ (see for instance [3]). We stress that we have assumed $\alpha_{i} \in \mathcal{K}$ instead of $\alpha_{i} \in$ $\mathcal{P}$ without any loss of generality due to the necessity result in [7] for the iISS feedback interconnection ${ }^{1}$. The following is a result in [9], which is referred to as the iISS small-gain condition in this paper.

Theorem 1: Suppose that Assumption 1 holds and that there exist $\omega_{1}, \omega_{2} \in \mathcal{K}_{\infty}$ satisfying

$$
\begin{aligned}
\alpha_{1}^{-1} \circ\left(\mathbf{I d}+\omega_{1}\right) \circ \sigma_{1} \circ \alpha_{2}^{-1} \circ\left(\mathbf{I d}+\omega_{2}\right) \circ \sigma_{2}(s) & \leq s, \\
\forall s & \in \mathbb{R}_{+} .
\end{aligned}
$$

Then, the following statements hold true:

(i) For $r(t) \equiv 0$, the system (1) is GAS.

(ii) If it holds that

$$
\left\{\lim _{s \rightarrow \infty} \alpha_{i}(s)=\infty \vee \lim _{s \rightarrow \infty} \sigma_{3-i}(s)<\infty\right\}, i=1,2,
$$

then the system (1) is iISS with respect to input $r$ and state $x$.

It is stressed that the small-gain condition (4) implicitly requires that

$$
\lim _{s \rightarrow \infty} \alpha_{2}(s)=\infty \vee \lim _{s \rightarrow \infty} \alpha_{2}(s)>\lim _{s \rightarrow \infty} \sigma_{2}(s) .
$$

The property (6) implies that $\Sigma_{2}$ is ISS with respect to its feedback input $x_{1}$ (see for instance [15], [3]). On the other hand, $\Sigma_{1}$ does not have to be ISS with respect to its feedback input $x_{2}$. The small-gain condition for iISS subsystems indicates that the interconnection is stable if the stability property of one subsystem, $\Sigma_{2}$, is strong enough to compensate the "weak stability" of the other subsystem, $\Sigma_{1}$. Due to this asymmetry, we need to select or interchange the indices " 1 " and " 2 " so that (4) holds when iISS subsystems are involved.

The necessity of the condition (6) for stability of the interconnected system is investigated in [9], which is summarized as follows:

Theorem 2: Suppose that $\alpha_{i} \in \mathcal{O}(1)$ and $\sigma_{i} \in \mathcal{O}(>0)$ are given for $i=1,2$. Then, the following statements hold true:

\footnotetext{
${ }^{1}$ In the case of cascade, assuming $\alpha_{i} \in \mathcal{K}$ is not necessary [4], [5].
}

(i) The system (1) with $r(t) \equiv 0$ is GAS for all subsystems satisfying Assumption 1 only if

$$
\lim _{s \rightarrow \infty} \alpha_{j}(s) \geq \lim _{s \rightarrow \infty} \sigma_{j}(s)
$$

holds for at least one of $j \in\{1,2\}$.

(ii) The system (1) is ISS with respect to input $r$ and state $x$ for all subsystems satisfying Assumption 1 only if

$$
\lim _{s \rightarrow \infty} \alpha_{j}(s)=\infty \vee \lim _{s \rightarrow \infty} \alpha_{j}(s)>\lim _{s \rightarrow \infty} \sigma_{j}(s)
$$

holds for at least one of $j \in\{1,2\}$.

Without any loss of generality, we can take $j=2$ for (7) and (8). This convention is used in the rest of this paper. The above theorem does not exactly state that (6) is necessary for the iISS of the interconnection. The difference between (6) and (7) is the equality. Taking into account the necessity of (6) for the ISS property, the key idea of this paper is to directly make use of the property (6) for proving Theorem 1 in order to interpret the "iISS" small-gain theorem as the combination of "a transient response" and "the ISS smallgain dynamics". In other words, we do not consider the case of

$$
\infty>\lim _{s \rightarrow \infty} \alpha_{2}(s)=\lim _{s \rightarrow \infty} \sigma_{2}(s)
$$

since it prevents us from using the ISS small-gain argument in the presence of the external signal $r$. Anyway, note that the case (9) is incompatible with the small gain condition (4) if $\omega_{1}, \omega_{2} \in \mathcal{K}_{\infty}$.

Remark 1: In the small-gain condition (4), this paper employs amplification factors $\omega_{1}, \omega_{2}$ which are of class $\mathcal{K}_{\infty}$. As shown in [9], these factors do not have to be class $\mathcal{K}_{\infty}$ functions in the case of GAS. Nevertheless, it can be verified that, under the condition (6), the stability theorem proposed in [9] requires the existence of $\omega_{1}, \omega_{2} \in \mathcal{K}_{\infty}$ satisfying (4) except when $\lim _{s \rightarrow \infty} \alpha_{2}(s)=\lim _{s \rightarrow \infty} \sigma_{2}(s)=$ $\lim _{s \rightarrow \infty} \alpha_{1}(s)=\lim _{s \rightarrow \infty} \sigma_{1}(s)=\infty$ holds. However, this case corresponds to the interconnection of ISS subsystems, which is already widely addressed in the literature and is not the purpose of the present paper. Therefore, we consider $\omega_{1}$, $\omega_{2} \in \mathcal{K}_{\infty}$ for (4) without any loss of generality as long as iISS subsystems are concerned.

Remark 2: The necessary conditions in Theorem 2 was proved for $\alpha_{i} \in \mathcal{O}(>1)$ in [9]. It can be verified that $\alpha_{i} \in$ $\mathcal{O}(>1)$ can be replaced by $\alpha_{i} \in \mathcal{O}(1)$ for supply rates given as functions of $V_{1}$ and $V_{2}$ as in Assumption 1.

\section{Systems Without InPUT: GAS}

This section considers the interconnected system (1) in the absence of the external signals, i.e., $r(t) \equiv 0$, and demonstrates Item $(i)$ of Theorem 1 by means of a transient response plus the ISS small-gain argument. Assume that (4) is satisfied for some $\omega_{1}, \omega_{2} \in \mathcal{K}_{\infty}$. Suppose for the time being that

$$
\lim _{s \rightarrow \infty} \alpha_{1}(s)<\infty \wedge \lim _{s \rightarrow \infty} \alpha_{1}(s) \leq \lim _{s \rightarrow \infty} \sigma_{1}(s) .
$$


Then, the small-gain condition (4) implies that there exists a positive constant $\sigma_{2}^{\max }$ such that

$$
\lim _{s \rightarrow \infty} \sigma_{2}(s) \leq \sigma_{2}^{\max }<\infty .
$$

Since $\sigma_{2}^{\max }$ is independent of $x_{1}$, the dissipation inequality (3) of $\Sigma_{2}$ and the property (6) implied by (4) guarantee that the state $x_{2}(t)$ is bounded and eventually enters the forward invariant set

$$
\mathbf{U}_{2}:=\left\{x_{2} \in \mathbb{R}^{n_{2}}: V_{2}\left(x_{2}\right) \leq \lim _{s \rightarrow \infty} \alpha_{2}^{-1} \circ \sigma_{2}(s)\right\} .
$$

In fact, there exists $\delta>0$ such that $\dot{V}_{2} \leq-\delta$ holds for all $x_{2} \notin \mathbf{U}_{2}$. Let $T \geq 0$ be such that

$$
x_{2}(t) \in \mathbf{U}_{2}, \quad \forall t \geq T
$$

Note that $T$ is finite, and that the state $x_{1}(t)$ is bounded over the time interval $[0, T]$ since $\Sigma_{1}$ is iISS with respect to $x_{2}$. The small-gain condition (4) proves that the dissipation inequality (2) of $\Sigma_{1}$ becomes an ISS property when the input $x_{2}$ is restricted to $\mathbf{U}_{2}$. Indeed, the condition (4) guarantees that

$$
\begin{aligned}
\sup _{x_{2} \in \mathbf{U}_{2}} \sigma_{1}\left(V_{2}\left(x_{2}\right)\right) & \leq \lim _{s \rightarrow \infty} \sigma_{1} \circ \alpha_{2}^{-1} \circ \sigma_{2}(s) \\
& \leq \lim _{s \rightarrow \infty}\left(\mathbf{I d}+\omega_{1}\right)^{-1} \circ \alpha_{1}(s) .
\end{aligned}
$$

Hence, we obtain from (2) and (3) that

$$
\begin{aligned}
& V_{1}\left(x_{1}\right) \geq \alpha_{1}^{-1} \circ\left(\mathbf{I d}+\omega_{1}\right) \circ \sigma_{1}\left(V_{2}\left(x_{2}\right)\right) \wedge x_{2} \in \mathbf{U}_{2} \\
& \quad \Rightarrow \dot{V}_{1}\left(x_{1}\right) \leq-\left(\mathbf{I d}-\left(\mathbf{I d}+\omega_{1}\right)^{-1}\right) \circ \alpha_{1}\left(V_{1}\left(x_{1}\right)\right) \\
& V_{2}\left(x_{2}\right) \geq \alpha_{2}^{-1} \circ\left(\mathbf{I d}+\omega_{2}\right) \circ \sigma_{2}\left(V_{1}\left(x_{1}\right)\right) \\
& \quad \Rightarrow \dot{V}_{2}\left(x_{2}\right) \leq-\left(\mathbf{I d}-\left(\mathbf{I d}+\omega_{2}\right)^{-1}\right) \circ \alpha_{2}\left(V_{2}\left(x_{2}\right)\right) .
\end{aligned}
$$

Note that $\mathbf{I d}-\left(\mathbf{I d}+\omega_{i}\right)^{-1} \in \mathcal{K}_{\infty}$ since $\left(\mathbf{I d}-\left(\mathbf{I d}+\omega_{i}\right)^{-1}\right) \circ$ $\left(s+\omega_{i}(s)\right)=\omega_{i}(s)$. Due to (12) and (13), the convergence of $x(t)$ to the origin $x=0$ departing from any $x(T) \in \mathbb{R}^{n_{1}} \times \mathbf{U}_{2}$ at $t=T$ is ensured by the small-gain condition (4). For instance, we can follow the proof for the interconnection of the two ISS subsystems given in [11], [10].

Finally, in the case that (10) does not hold, that is

$$
\lim _{s \rightarrow \infty} \alpha_{1}(s)=\infty \vee \lim _{s \rightarrow \infty} \alpha_{1}(s)>\lim _{s \rightarrow \infty} \sigma_{1}(s),
$$

the subsystem $\Sigma_{1}$ is ISS with respect $x_{2}$ and we can invoke the ISS small-gain argument from $t=0$. This completes the proof of GAS, i.e. Item (i) of Theorem 1.

To sum up, the behavior before $t=T$ is a transient, and after $t=T$, the contractive dynamics kicks in since the iISS small-gain condition acts as the ISS small-gain condition in the domain the trajectories evolve. If $\Sigma_{1}$ is ISS, the transition time $T$ is zero.

Remark 3: In the absence of external signals, i.e. $r(t) \equiv$ 0 , a Lyapunov function establishing the GAS of the interconnected system can be constructed even when (9) holds. In fact, Theorem 1 in [9] derives such a Lyapunov function from a small-gain condition. The small-gain condition is in the form of (4). However, as already stressed, the amplification factors $\omega_{1}, \omega_{2}$ for GAS case are not necessarily of class $\mathcal{K}_{\infty}$ in the absence of the exogenous signal $r$. Although the above argument does not explicitly address (9), the observation of the transient plus the ISS small-gain dynamics still holds true. Notice that the ISS small-gain theorem applies to the case of

$$
\lim _{s \rightarrow \infty} \alpha_{1}(s)=\lim _{s \rightarrow \infty} \sigma_{1}(s) \wedge \text { Eq. (9) }
$$

directly since $V_{1}$ and $V_{2}$ become ISS Lyapunov functions of the individual subsystems. If

$$
\lim _{s \rightarrow \infty} \alpha_{1}(s)>\lim _{s \rightarrow \infty} \sigma_{1}(s) \wedge \text { Eq. (9) }
$$

holds, by virtue of $\alpha_{2}^{-1} \circ \sigma_{2} \in \mathcal{K}_{\infty}$, the argument given in this section can be used by switching the indices " 1 " and "2". The situation

$$
\lim _{s \rightarrow \infty} \alpha_{1}(s)<\lim _{s \rightarrow \infty} \sigma_{1}(s) \wedge \text { Eq. (9) }
$$

is excluded by Theorem 5 (i) in [9]. Therefore, for the GAS case (i.e., for $r(t) \equiv 0$ ), the interpretation of the transient plus the ISS small-gain dynamics is valid whenever

$$
\lim _{s \rightarrow \infty} \alpha_{2}(s) \geq \lim _{s \rightarrow \infty} \sigma_{2}(s)
$$

\section{SySTEMS WITH INPUT: iISS}

This section proves Item (ii) of Theorem 1 under particular assumptions. The property (6) implied by the small-gain condition (4) again plays a key role in implementing the idea of a transient plus the ISS small-gain argument. The proof consists of two parts. One is to verify that the system (1) is 0 -GAS (that is, GAS when $r(t) \equiv 0$ ). The other part is to establish the Bounded Energy Frequently Bounded State (BEFBS) property of the system (1). It is shown in [2] that the combination of the above two properties is equivalent to the iISS property of the system (1). Since the 0-GAS has been proved in the previous section, this section is devoted to the BEFBS property.

First, notice that $\lim _{s \rightarrow \infty} \alpha_{i}(s)>\lim _{s \rightarrow \infty} \sigma_{i}(s)$ does not guarantee the ISS property of $\Sigma_{i}$ with respect to input $\left(x_{3-i}, r_{i}\right)$ since $\lim _{s \rightarrow \infty} \sigma_{r i}(s)$ can anyway be larger than $\lim _{s \rightarrow \infty} \alpha_{i}(s)$. In fact, when there exists $i \in\{1,2\}$ such that $\lim _{s \rightarrow \infty} \alpha_{i}(s)<\infty$ holds, the previously existing results only show the iISS of the interconnected system [6], [9]. Hence, in contrast to the GAS case, the condition $\lim _{s \rightarrow \infty} \alpha_{2}(s)>\lim _{s \rightarrow \infty} \sigma_{2}(s)$ is not sufficient for resorting to the ISS small-gain argument in the presence of external inputs. In order to make use of the small-gain argument of ISS-type, we assume the following in addition to (6).

Assumption 2: The following properties hold:

$$
\begin{gathered}
\lim _{s \rightarrow \infty}\left\{\sigma_{1} \circ \alpha_{2}^{-1} \circ\left(\mathbf{I d}+\omega_{2}^{-1}\right) \circ \sigma_{r 2}(s)+\sigma_{r 1}(s)\right\} \\
\leq \lim _{s \rightarrow \infty}\left(\mathbf{I d}-\left(\mathbf{I d}+\omega_{1}\right)^{-1}\right) \circ \alpha_{1}(s) \\
\lim _{s \rightarrow \infty} \alpha_{2}(s)=\infty \vee \\
\lim _{s \rightarrow \infty} \alpha_{2}(s)>\lim _{s \rightarrow \infty}\left(\mathbf{I d}-\left(\mathbf{I d}+\omega_{2}\right)^{-1}\right)^{-1} \circ \sigma_{r 2}(s) .
\end{gathered}
$$


Note that $\mathbf{I d}+\omega_{2}^{-1} \in \mathcal{K}_{\infty}$. We are now ready to prove the BEFBS property of the system (1). Due to (19), there exists $\beta \in \mathcal{K}_{\infty}$ such that

$$
\begin{aligned}
& \lim _{s \rightarrow \infty} \alpha_{2}(s)=\infty \vee \\
& \lim _{s \rightarrow \infty} \alpha_{2}(s) \geq \lim _{s \rightarrow \infty}\left((\mathbf{I d}+\beta)^{-1}-\left(\mathbf{I d}+\omega_{2}\right)^{-1}\right)^{-1} \circ \sigma_{r 2}(s) .
\end{aligned}
$$

By virtue of (20) and

$$
\lim _{s \rightarrow \infty} \alpha_{2}(s) \geq \lim _{s \rightarrow \infty}\left(\mathbf{I d}+\omega_{2}\right) \circ \sigma_{2}(s)
$$

implied by (4), the property

$$
\lim _{s \rightarrow \infty} \alpha_{2}(s) \geq \lim _{s \rightarrow \infty}(\mathbf{I d}+\beta) \circ\left(\sigma_{2}(s)+\sigma_{r 2}(s)\right)
$$

holds since

$$
\begin{aligned}
& \lim _{s \rightarrow \infty} \sigma_{2}(s)+\lim _{s \rightarrow \infty} \sigma_{r 2}(s) \leq \\
& \quad \lim _{s \rightarrow \infty}\left(\left(\mathbf{I d}+\omega_{2}\right)^{-1}+(\mathbf{I d}+\beta)^{-1}-\left(\mathbf{I d}+\omega_{2}\right)^{-1}\right) \circ \alpha_{2}(s)
\end{aligned}
$$

in the case of $\lim _{s \rightarrow \infty} \alpha_{2}(s)<\infty$. Suppose that

$$
\lim _{s \rightarrow \infty} \alpha_{1}(s)<\infty \vee \lim _{s \rightarrow \infty} \alpha_{2}(s)<\infty
$$

holds since the standard ISS small-gain theorem applies to the case of $\lim _{s \rightarrow \infty} \alpha_{1}(s)=\infty \wedge \lim _{s \rightarrow \infty} \alpha_{2}(s)=\infty$. If $\lim _{s \rightarrow \infty} \alpha_{1}(s)<\infty$ holds, the property (5) implies $\lim _{s \rightarrow \infty} \sigma_{2}(s)<\infty$. In the case of $\lim _{s \rightarrow \infty} \alpha_{2}(s)<\infty$, the property (6) yields $\lim _{s \rightarrow \infty} \sigma_{2}(s)<\infty$. Thus, when (22) is satisfied, there exist $\sigma_{2}^{\max }>0$ such that (11) holds. If $\lim _{s \rightarrow \infty} \sigma_{r 2}(s)<\infty$ holds, the dissipation inequality (3) of $\Sigma_{2}$ guarantees that the state $x_{2}(t)$ which is bounded enters the set

$$
\begin{aligned}
\mathbf{U}_{D 2}:= & \left\{x_{2} \in \mathbb{R}^{n_{2}}:\right. \\
& \left.V_{2}\left(x_{2}\right) \leq \lim _{s \rightarrow \infty} \alpha_{2}^{-1} \circ\left\{\sigma_{2}(s)+\sigma_{r 2}(s)\right\}\right\}
\end{aligned}
$$

in a finite time and remains there. Here, (21) is used. Let $T \geq 0$ be such that

$$
x_{2}(t) \in \mathbf{U}_{D 2}, \quad \forall t \geq T .
$$

Note that the state $x_{1}(t)$ is also bounded for the time interval $[0, T]$ since $\Sigma_{1}$ is iISS with respect to input $\left(x_{2}, r_{1}\right)$ and state $x_{1}$. Combining (4) and $x_{2} \in \mathbf{U}_{D 2}$ yields

$$
\begin{aligned}
\sup _{x_{2} \in \mathbf{U}_{D 2}} \sigma_{1}\left(V_{2}\left(x_{2}\right)\right) \leq \lim _{s \rightarrow \infty} \sigma_{1} \circ \alpha_{2}^{-1} \circ\left\{\sigma_{2}(s)+\sigma_{r 2}(s)\right\} \\
\leq \lim _{s \rightarrow \infty} \sigma_{1} \circ \alpha_{2}^{-1} \circ\left(\mathbf{I d}+\omega_{2}\right) \circ \sigma_{2}(s) \\
\quad+\lim _{s \rightarrow \infty} \sigma_{1} \circ \alpha_{2}^{-1} \circ\left(\mathbf{I d}+\omega_{2}^{-1}\right) \circ \sigma_{r 2}(s) \\
\leq \lim _{s \rightarrow \infty}\left(\mathbf{I d}+\omega_{1}\right)^{-1} \circ \alpha_{1}(s) \\
\quad+\lim _{s \rightarrow \infty} \sigma_{1} \circ \alpha_{2}^{-1} \circ\left(\mathbf{I d}+\omega_{2}^{-1}\right) \circ \sigma_{r 2}(s) .
\end{aligned}
$$

To derive the second inequality, the two cases separated by $\omega_{2} \circ \sigma_{2}(s) \geq \sigma_{r 2}(s)$ and $\omega_{2} \circ \sigma_{2}(s)<\sigma_{r 2}(s)$ are combined. Hence, from (2) it follows that, for all $x_{2} \in \mathbf{U}_{D 2}$,

$$
\begin{gathered}
\dot{V}_{1}\left(x_{1}\right) \leq-\alpha_{1}\left(V_{1}\left(x_{1}\right)\right)+\lim _{s \rightarrow \infty}\left(\mathbf{I d}+\omega_{1}\right)^{-1} \circ \alpha_{1}\left(V_{1}\left(x_{1}\right)\right) \\
+\lim _{s \rightarrow \infty} \sigma_{1} \circ \alpha_{2}^{-1} \circ\left(\mathbf{I d}+\omega_{2}^{-1}\right) \circ \sigma_{r 2}(s)+\sigma_{r 1}\left(\left|r_{1}\right|\right) .
\end{gathered}
$$

Thus, the property (18) implies the bounded-input boundedstate property over $t \in[T, \infty)$ provided that $x_{2}(T) \in$ $\mathbf{U}_{D 2}$, which holds true in view of (23). This together with the boundedness of $x_{1}(t)$ and $x_{2}(t)$ in the interval $[0, T)$ establishes the BEFBS property for all $x(0) \in \mathbb{R}^{n}$.

If $\lim _{s \rightarrow \infty} \sigma_{r 2}(s)=\infty$ holds, the property (18) yields

$$
\begin{aligned}
\lim _{s \rightarrow \infty}\left\{\sigma_{1}(s)+\sigma_{r 1}(s)\right\} & \leq \lim _{s \rightarrow \infty}\left(\mathbf{I d}-\left(\mathbf{I d}+\omega_{1}^{-1}\right)\right) \circ \alpha_{1}(s) \\
& \leq \lim _{s \rightarrow \infty} \alpha_{1}(s) .
\end{aligned}
$$

Hence, the BEFBS property with respect to input $r$ and state $x_{1}$ follows from (2). Due to the fact that $\lim _{s \rightarrow \infty} \alpha_{2}(s)=\infty$ and $V_{1}\left(x_{1}\right) \leq B<\infty$ with a constant $B$ guaranteed by (25), the BEFBS property with respect to input $r$ and state $x_{2}$ follows from (3). This completes the proof of Item (ii) of Theorem 1 .

Thus, even in the presence of the external signal $r$, the behavior up to $t=T$ can be considered as a transient. After $t=T$, the bounded-input bounded-state property takes effect since the iISS small-gain condition acts as the ISS small-gain condition in the domain the trajectories evolve. The bounded-input bounded-state property preceded by the transient implies the BEFBS property. If $\Sigma_{1}$ is ISS, the transition time $T$ is equal to zero.

\section{ANOTHER FORMULATION OF iISS}

The idea of the reduction to the ISS small-gain argument in the presence of an iISS subsystem can be seen in a more compact manner if one uses dissipation inequalities of another type for the iISS property of the individual subsystems. To this end, in this section, we replace Assumptions 1 and 2 with the following two assumptions:

Assumption 3: For each $i \in\{1,2\}$, there exist a continuously differentiable positive definite and radially unbounded function $V_{i}: \mathbb{R}^{n_{i}} \rightarrow \mathbb{R}_{+}$and class $\mathcal{K}$ functions $\alpha_{i}, \sigma_{i}, \sigma_{r i}$ such that

$$
\begin{aligned}
& \dot{V}_{1}\left(x_{1}\right) \leq-\alpha_{1}\left(V_{1}\left(x_{1}\right)\right)+\max \left\{\sigma_{1}\left(V_{2}\left(x_{2}\right)\right), \sigma_{r 1}\left(\left|r_{1}\right|\right)\right\} \\
& \dot{V}_{2}\left(x_{2}\right) \leq-\alpha_{2}\left(V_{2}\left(x_{2}\right)\right)+\max \left\{\sigma_{2}\left(V_{1}\left(x_{1}\right)\right), \sigma_{r 2}\left(\left|r_{2}\right|\right)\right\}
\end{aligned}
$$

hold for all $r \in \mathcal{U}$ along the trajectories $x(t)$ of (1).

Assumption 4: The following properties hold:

$$
\begin{aligned}
& \lim _{s \rightarrow \infty} \alpha_{1}(s)=\infty \vee \lim _{s \rightarrow \infty} \alpha_{1}(s)>\lim _{s \rightarrow \infty} \sigma_{r 1}(s) \\
& \lim _{s \rightarrow \infty} \alpha_{2}(s)=\infty \vee \lim _{s \rightarrow \infty} \alpha_{2}(s)>\lim _{s \rightarrow \infty} \sigma_{r 2}(s) .
\end{aligned}
$$

When the interconnection of two iISS subsystems is defined with Assumption 3 in Theorem 1, we are able to achieve the reduction to the transient plus the ISS smallgain argument under Assumption 4 which looks simpler than Assumption 2. The rest of this section gives a proof demonstrating this fact for Item (ii) of Theorem 1.

Since the 0-GAS property is proved in Section III, we shall prove the BEFBS property of the system (1). As in Section IV, we can assume (22). The properties (5) and (6) ensure the existence of $\sigma_{2}^{\max }>0$ satisfying (11). Suppose 
that $\lim _{s \rightarrow \infty} \sigma_{r 2}(s)<\infty$. Due to (11), (29) and (6), the dissipation inequality (27) of $\Sigma_{2}$ guarantees that the state $x_{2}(t)$ which is bounded enters the set

$$
\mathbf{U}_{2}:=\left\{x_{2} \in \mathbb{R}^{n_{2}}: V_{2}\left(x_{2}\right) \leq \lim _{s \rightarrow \infty} \alpha_{2}^{-1} \circ \sigma_{2}(s)\right\}
$$

in a finite time $T$ and stays there. When $\lim _{s \rightarrow \infty} \sigma_{r 2}(s)=\infty$ holds, the same property holds with $T$ which satisfies $T<\infty$ for $\left|r_{2}\right|<\infty$. The state $x_{1}(t)$ is also bounded for the time interval $[0, T]$ since $\Sigma_{1}$ is iISS with respect to input $\left(x_{2}, r_{1}\right)$ and state $x_{1}$. The small-gain condition (4) yields

$$
\begin{aligned}
\sup _{x_{2} \in \mathbf{U}_{2}} \sigma_{1}\left(V_{2}\left(x_{2}\right)\right) & \leq \lim _{s \rightarrow \infty} \sigma_{1} \circ \alpha_{2}^{-1} \circ \sigma_{2}(s) \\
& \leq \lim _{s \rightarrow \infty}\left(\mathbf{I d}+\omega_{1}\right)^{-1} \circ \alpha_{1}(s)
\end{aligned}
$$

From (26) it follows that, for all $x_{2} \in \mathbf{U}_{2}$,

$$
\begin{aligned}
\dot{V}_{1}\left(x_{1}\right) \leq & -\alpha_{1}\left(V_{1}\left(x_{1}\right)\right)+ \\
& \max \left\{\lim _{s \rightarrow \infty}\left(\mathbf{I d}+\omega_{1}\right)^{-1} \circ \alpha_{1}(s), \sigma_{r 1}\left(\left|r_{1}\right|\right)\right\} .
\end{aligned}
$$

Thus, the assumption (28) leads us to the BEFBS property with respect to input $r$ and state $x_{1}$ in the interval of $t \in[T, \infty)$ for the initial conditions $x_{2}(T) \in \mathbf{U}_{2}$. Since we have $x_{2}(t) \in \mathbf{U}_{2}$ for all $t \in[T, \infty)$, the BEFBS property with respect to input $r$ and state $x_{2}$ also holds in the interval of $t \in[T, \infty)$ for the initial condition $x_{2}(T) \in \mathbf{U}_{2}$. These properties together with the boundedness of $x_{1}(t)$ and $x_{2}(t)$ in the interval $[0, T)$ establish the BEFBS property with respect to input $r$ and state $x$ in the interval of $t \in[0, \infty)$ for all $x(0) \in \mathbb{R}^{n}$. This completes the proof of Item (ii) of Theorem 1.

Assumption 1 and Assumption 3 are qualitatively equivalent in the sense that $\sigma_{i}+\sigma_{r i} \leq \max \left\{2 \sigma_{i}, 2 \sigma_{r i}\right\} \leq$ $2 \sigma_{i}+2 \sigma_{r i}$. We can consider other variants of dissipation inequalities for iISS. Although the coefficients appearing in the transformation between two representations result in conservativeness in different forms, the essence of imposing the constraint on the external inputs for the reduction to the ISS small-gain argument remains the same.

Remark 4: As already stressed, the difficulty in establishing the iISS via the transient plus the ISS small-gain dynamics arises when the effect of $r_{i}$ 's is larger than the contribution of $\alpha_{i}$ 's. Both Assumption 1 and Assumption 3 allow the magnitude of $\sigma_{r i}$ 's to be arbitrarily large. In order to make the ISS small-gain argument work, the undesirably large effect of $r_{i}$ 's is avoided by Assumptions 2 and 4 . In short, $\sigma_{r i}$ 's are required to be sufficiently small in this paper. It is worth noticing that the pair of ISS with respect to small inputs and forward completeness does not always imply iISS. Indeed, one can construct a forward complete non-iISS system of the form $\dot{x}=f(x)+g(x, r) r$ which is ISS with respect to small inputs by modifying the technique proposed in [3, Section V]. In the presence of arbitrarily large $\sigma_{r i}$ 's, removing Assumptions 2 and 4 is inherently difficult.

Remark 5: Neither the pair (18)-(19) nor the pair (28)(29) is necessary for establishing the iISS property of the

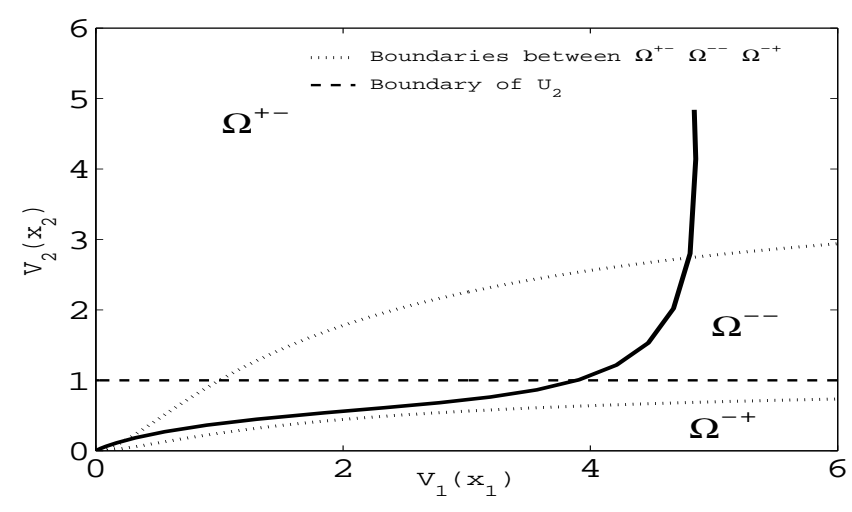

Fig. 1. A trajectory on the $\left(V_{1}, V_{2}\right)$-plane without disturbances.

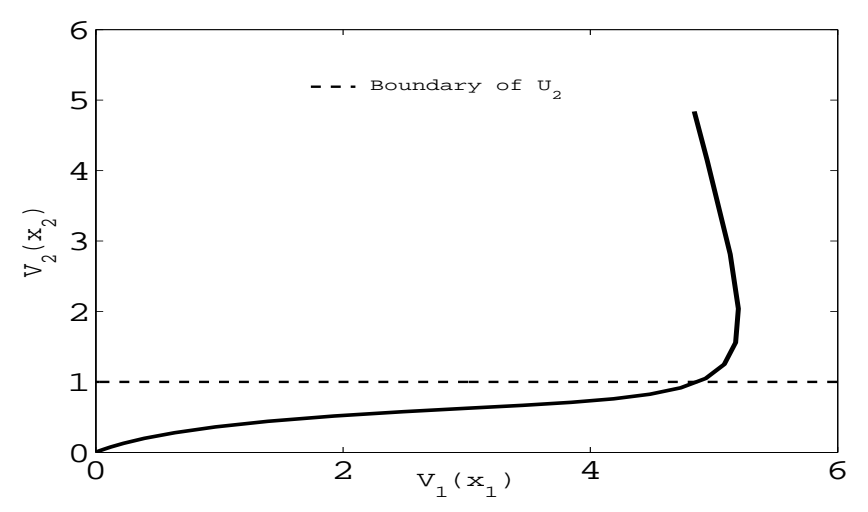

Fig. 2. A trajectory on the $\left(V_{1}, V_{2}\right)$-plane with a disturbance.

interconnection of iISS subsystems. For example, in the case where $\alpha_{1}=\sigma_{2}, \alpha_{2}=d \sigma_{1}$ with $\sigma_{r 1}, \sigma_{r 2} \in \mathcal{K}_{\infty}$ and some $d>1$, the function $V=V_{1}+V_{2}(1+1 / d) / 2$ is an iISS Lyapunov function, thus immediately proving the iISS of the interconnection. In contrast to the approach followed in this paper, this case is covered by the iISS small-gain theorems proposed in [6], [9]. Therefore, the approach based on the ISS small-gain argument plus the transient is more restrictive than the direct iISS small-gain approach.

\section{AN EXAMPLE}

Consider the interconnected system described by

$$
\begin{aligned}
& \dot{x}_{1}=-\frac{x_{1}}{1+x_{1}^{2}}+\frac{x_{1}}{2\left(1+x_{1}^{2}\right)}\left(x_{2}+r_{1}\right) \\
& \dot{x}_{2}=-x_{2}+\frac{x_{1}^{2}}{1+x_{1}^{2}}
\end{aligned}
$$

This pair satisfies the dissipation inequalities

$$
\begin{aligned}
& \dot{V}_{1}\left(x_{1}\right) \leq-\frac{2 V_{1}\left(x_{1}\right)}{1+V_{1}\left(x_{1}\right)}+\sqrt{V_{2}\left(x_{2}\right)}+\left|r_{1}\right| \\
& \dot{V}_{2}\left(x_{2}\right) \leq-V_{2}\left(x_{2}\right)+\left(\frac{V_{1}\left(x_{1}\right)}{1+V_{1}\left(x_{1}\right)}\right)^{2}
\end{aligned}
$$

for $V_{1}\left(x_{1}\right)=x_{1}^{2}$ and $V_{2}\left(x_{2}\right)=x_{2}^{2}$. Note that the upper bounds in (33) and (34), i.e., the supply rates, may not be completely tight. The subsystem $\Sigma_{1}$ is not ISS with respect to input $x_{2}$, and it is only iISS. The trajectory of (31)-(32) for the initial condition $x(0)=[2.2,2.2]^{T}$ is plotted on the 
$\left(V_{1}, V_{2}\right)$-plane in Fig. 1 for $r_{1}(t) \equiv 0$. Figure 1 also depicts the following sets:

$$
\begin{gathered}
\Omega^{+-}:=\left\{\left(V_{1}, V_{2}\right) \in \mathbb{R}_{+}^{2}: \alpha_{1}\left(V_{1}\right) \leq \sigma_{1}\left(V_{2}\right)\right. \\
\left.\wedge \alpha_{2}\left(V_{2}\right) \geq \sigma_{2}\left(V_{1}\right)\right\} \\
\Omega^{--}:=\left\{\left(V_{1}, V_{2}\right) \in \mathbb{R}_{+}^{2}: \alpha_{1}\left(V_{1}\right) \geq \sigma_{1}\left(V_{2}\right)\right. \\
\left.\wedge \alpha_{2}\left(V_{2}\right) \geq \sigma_{2}\left(V_{1}\right)\right\} \\
\Omega^{-+}:=\left\{\left(V_{1}, V_{2}\right) \in \mathbb{R}_{+}^{2}: \alpha_{1}\left(V_{1}\right) \geq \sigma_{1}\left(V_{2}\right)\right. \\
\left.\wedge \alpha_{2}\left(V_{2}\right) \leq \sigma_{2}\left(V_{1}\right)\right\} .
\end{gathered}
$$

The boundaries of these sets are not necessarily the nullclines of (31) and (32) owing to the lack of tightness in the dissipation inequalities (33) and (34). Two phases are observed in Fig. 1. The first phase is the transient evolving outside $\mathbf{U}_{2}$ for which the trajectory heads. The second phase is the trajectory converging to the origin without leaving $\mathbf{U}_{2}$. Once the trajectory enters the positively invariant set $\mathbf{U}_{2}$, the dynamic is governed by the ISS small-gain condition as discussed in Section III. It is also seen in Fig. 1 near the origin that the set $\Omega^{--}$is too narrow to be an invariant set because of the gaps in the dissipation inequalities. Figure 2 shows the response for the same initial condition in the presence of disturbances $r_{1}(t)=1.8 /(2+t)$ and $r_{2}(t) \equiv 0$. The trajectory is bounded and moves toward the set $\mathbf{U}_{2}$ which becomes positively invariant again. Since the iISS small-gain condition acts as the ISS small-gain condition in $\mathbf{U}_{2}$, we see that the trajectory converges to the origin. It conforms to the converging-input converging-state of the ISS property. The boundedness and the converging property agree with the iISS property for the entire $t \geq 0$ which is established in Section IV.

\section{CONCluding Remarks}

This paper has investigated the iISS small-gain theorem developed in [6], [9] in order to give it a trajectory-based interpretation linking with the contractive mechanism of the ISS small-gain theorem. A preceding study has proved that the interconnection of two iISS systems is stable if one subsystem is ISS with respect to its feedback input strongly enough to compensate the weak stability of the other iISS subsystem. By making use of this fact, this paper has shown that the behavior of the interconnected system can be split into two phases. In the first phase, roughly, the trajectory of the ISS subsystem behaves almost independently of the other iISS subsystem and this phase lasts until the trajectory of the ISS subsystem enters a neighborhood $\mathbf{U}_{2}$ of the origin with a certain radius. In this phase, the behavior of the merely iISS subsystem is almost a free response. In the second phase, the interaction between the two subsystems takes effect and the contractive behavior of the whole state vector occurs since the small-gain constraint plays the role of the ISS small-gain condition in $\mathbf{U}_{2}$. This observation would be practically useful in designing and analyzing the dynamics of nonlinear control systems based on the iISS small-gain theorem. It is worth stressing that the above interpretation is not always applicable. The external signals are not allowed to be large either as in (18)-(19) or (28)(29). These assumptions ensure that the transient response actually dies in finite time which allows us to make use of the "ISS" small-gain argument for the subsequent behavior in dealing with "iISS" subsystems. There are interconnected systems which violate these assumptions and can anyway be proved to be iISS with respect to the external signals by constructing Lyapunov functions as in [6], [9].

After the submission of this paper, the authors became aware of an independent study [12] verifying input-to-output stability and computing the gain of interconnected systems. It uses a similar idea of combining a small-gain argument with a transient under the assumption that an estimate of trajectories is somehow available during the time period when the ISS-type small-gain criterion is invalid. In contrast, this paper here does not assume anything more than the standard iISS dissipation inequalities of subsystems, which would be less demanding than the time embedded trajectory estimate. Nevertheless, it is remarkable that an abstract model is employed in [12] for covering a considerably broad class of systems at the price of some complexities in the stability criterion.

\section{REFERENCES}

[1] D. Angeli and A. Astolfi, "A tight small gain theorem for not necessarily ISS systems," Syst. Control Lett., vol. 56, pp. 87-91, 2007.

[2] D. Angeli, B. Ingalls , E.D. Sontag and Y. Wang, "Separation principles for input-output and integral-input-to-state stability," SIAM J. Control Optim., vol. 43, pp. 256-276, 2004.

[3] D. Angeli, E.D. Sontag and Y. Wang, "A characterization of integral input-to-state stability," IEEE Trans. Autom. Control, vol. 45, pp. 10821097,2000

[4] M. Arcak, D. Angeli, and E. Sontag, "A unifying integral ISS framework for stability of nonlinear cascades," SIAM J. Control Optim., vol. 40, pp. 1888-1904, 2002.

[5] A. Chaillet, and D. Angeli, "Integral input to state stable systems in cascade," Syst. Control Lett., 57, pp. 519-527, 2008.

[6] H. Ito, "State-dependent scaling problems and stability of interconnected iISS and ISS systems," IEEE Trans. Autom. Control, vol. 51, pp.1626-1643, 2006.

[7] H. Ito, "A Lyapunov approach to integral input-to-state stability of cascaded systems with external signals," Proc. 47th IEEE Conf. Decision and Control, pp. 628-633, 2008.

[8] H. Ito, R.A. Freeman and A. Chaillet, "A two-phase interpretation of the iISS small-gain theorem", SICE 10th Annual Conf. Control Systems, 166-1-1(6 pages), March, 2010.

[9] H. Ito and Z.-P. Jiang, "Necessary and sufficient small gain conditions for integral input-to-state stable systems: A Lyapunov perspective," IEEE Trans. Autom. Contr, vol.54, pp.2389-2404, 2009.

[10] Z-P. Jiang, I. Mareels, and Y. Wang, "A Lyapunov formulation of the nonlinear small-gain theorem for interconnected ISS systems," Automatica, vol. 32, pp. 1211-1215, 1996.

[11] Z-P. Jiang, A.R. Teel, and L. Praly. "Small-gain theorem for ISS systems and applications," Mathe. Contr. Signals and Syst., vol. 7, pp.95-120, 1994.

[12] I. Karafyllis and Z.-P. Jiang, "New results in trajectory-based smallgain with application to the stabilization of a chemostat", Submitted, 2010, Available online: http://arxiv.org/abs/1002.4489.

[13] E.D. Sontag. "Smooth stabilization implies coprime factorization," IEEE Trans. Autom. Contr, vol. 34, pp.435-443, 1989.

[14] E.D. Sontag, "Comments on integral variants of ISS," Syst. Control Lett., vol. 34, pp. 93-100, 1998.

[15] E.D. Sontag, and Y. Wang. "On characterizations of input-to-state stability property," Syst.Control Lett.,24,pp.351-359,1995.

[16] A. Teel, "A nonlinear small gain theorem for the analysis of control systems with saturation," IEEE Trans. Automat. Control, vol. 41, pp. 1256-1270, 1996. 